\begin{tabular}{|c|c|}
\hline Title & A nisotropic Double-Network Hydrogels via Controlled Orientation of a Physical Sacrificial Network \\
\hline Author(s) & King, Daniel R.; Takahashi, Riku; Ikai, T akuma; Fukao, Kazuki; Kurokawa, T akayuki; Gong, Jian Ping \\
\hline Citation & $\begin{array}{l}\text { A CS applied polymer materials, 2(6), 2350-2358 } \\
\text { https://doi.org/10.1021/acsapm.0c00290 }\end{array}$ \\
\hline Issue Date & $2020-06-12$ \\
\hline Doc URL & http:/hdl.handle.net/2115/81827 \\
\hline Rights & $\begin{array}{l}\text { This document is the A ccepted Manuscript version of a Published Work that appeared in final form in ACS applied } \\
\text { polymer materials, copyright @2020 A merican Chemical Society after peer review and technical editing by the } \\
\text { publisher. To access the final edited and published work see 10.1021/acsapm.0c00290 }\end{array}$ \\
\hline Type & article (author version) \\
\hline File Information & Manuscript Resubmission_Final.pdf \\
\hline
\end{tabular}

Instructions for use 


\section{Anisotropic Double Network Hydrogels via Controlled Orientation of a Physical Sacrificial Network}

Daniel R. King ${ }^{1,2, *, \dagger}$, Riku Takahashi ${ }^{3,+, \$}$, Takuma Ikai ${ }^{3, \dagger}$, Kazuki Fukao $^{3}$, Takayuki Kurokawa ${ }^{1,2}$, \& Jian Ping Gong ${ }^{1,2,4 *}$

${ }^{1}$ Faculty of Advanced Life Science, Hokkaido University, Sapporo 001-0021, Japan.

${ }^{2}$ Global Station for Soft Matter, Global Institution for Collaborative Research and Education (GICoRE), Hokkaido University, Sapporo 001-0021, Japan.

${ }^{3}$ Graduate School of Life Science, Hokkaido University, Sapporo 001-0021, Japan.

${ }^{4}$ Institute for Chemical Reaction Design and Discovery (WPI-ICReDD), Hokkaido University, Sapporo, 001-0021, Japan.

${ }^{\$}$ Current Address: NTT Basic Research Laboratories, Bio-Medical Informatics Research Center, NTT Corporation, 3-1 Morinosato-Wakamiya, Atsugi, Kanagawa, 243-0198, Japan.

${ }^{\dagger}$ The authors contributed equally to this work

*Corresponding author: dking@sci.hokudai.ac.jp; gong@sci.hokudai.ac.jp

KEYWORDS: Hydrogels, Semi-rigid polymers, Composite materials, Double network gels, Anisotropic structures, Anisotropic mechanical properties 


\begin{abstract}
We report a method to create anisotropic double network (DN) hydrogels, through the controlled orientation of a physical sacrificial network. A crosslinked polyacrylamide hydrogel is synthesized from a solution containing a semi-rigid anionic polyelectrolyte. Subsequently, the gel is stretched to orient the semi-rigid polyelectrolyte, which does not relax in the stretched state due to the high contour length in comparison to the mesh size of the polyacrylamide network. The polyelectrolyte is then physically crosslinked with a multivalent cation, $\mathrm{ZrCl}_{2} \mathrm{O}$, to fix the anisotropy. Anisotropy was visualized by observing birefringence and quantified by small angle x-ray scattering (SAXS). By comparing the scattering in the oriented direction versus perpendicular to the oriented direction, a structural anisotropy factor was calculated. Uniaxial tensile testing was performed on samples of varying pre-stretch, both parallel and perpendicular to the stretching direction. The Young's modulus, fracture stress, fracture strain, and work of extension were characterized, and the resulting mechanical anisotropy was compared to the structural anisotropy factor. We find that the anisotropy of Young's modulus and fracture stress are directly controlled by the anisotropy of the sacrificial network, while fracture strain and work of extension show little influence from structural anisotropy. The results of this work demonstrate that pre-stretching of a physical sacrificial network is a controllable and simple method to create anisotropic DN hydrogels.
\end{abstract}




\section{INTRODUCTION}

Load-bearing soft tissues represent a unique type of soft matter: while containing water, they possess robust mechanical properties over a diverse spectrum of stiffnesses. ${ }^{1,2}$ Ranging from softest to hardest, some examples include skin, ${ }^{3}$ ligament, ${ }^{4-6}$ and cartilage. ${ }^{7-9}$ Interestingly, all of these materials possess anisotropic and hierarchical structures, which enables important functions. Ligaments are composed of aligned collagen fibrils within an extracellular matrix, providing high stiffness along with water content. Cartilage is also a soft material that contains collagen, but the orientation of the collagen fibrils changes spatially. Close to the bone, collagen is aligned perpendicular to the surface, providing high compression stiffness. Moving away from the bone, the collagen becomes increasingly isotropic, and then reverses close to the interface, eventually becoming aligned parallel to the surface. ${ }^{8}$ This parallel alignment provides stiffness against the tension experienced during motion. Creation of synthetic materials that possess similar controlled anisotropy and robust mechanical properties similar to biological tissues is a significant challenge.

Hydrogels are often compared to biological tissues because they contain large amounts of water. Yet simple hydrogels are brittle and weak, which has limited their application. Significant progress has been achieved towards improving the mechanical properties of hydrogels through the development of numerous toughening mechanisms. ${ }^{10-13}$ Among the many methods developed, double network (DN) gels stand out due to their combination of high modulus, toughness, and water content. ${ }^{11,14} \mathrm{DN}$ gels consist of an interpenetrating network structure, where the first network is rigid and brittle, and the second network is soft and stretchable. When deformed, covalent bonds in the first network fracture, dissipating energy. This fracture process permanently remodels the structure of the gel, and this dissipation process cannot be repeated.

Recently, we developed a new type of DN gel with a physical sacrificial network based on 
the semi-rigid polymer poly(2,2'-disulfonyl-4,4'-benzidine terephthalamide) (PBDT, Figure 1a(i)) reinforcing a crosslinked polyacrylamide (PAAm) matrix (Figure 1a(ii)). ${ }^{15}$ Polyelectrolytes are capable of physical crosslinking through the incorporation of multivalent metal ions. ${ }^{16-21}$ Because these bonds are capable of breaking and reforming, they represent a method to create a sacrificial network that can reversibly dissipate energy. In the reaction cell, PBDT was mixed with acrylamide monomer, a chemical crosslinker, and potassium persulfate (KPS) as an initiator. Thermal polymerization was carried out in air in a temperature-controlled heat box at $60^{\circ} \mathrm{C}$. After polymerization, the hydrogel was immersed in $\mathrm{ZrCl}_{2} \mathrm{O}$ solution. $\mathrm{ZrCl}_{2} \mathrm{O}$ forms a complex compound, $\left[\mathrm{Zr}(\mathrm{OH})_{2} \cdot 4 \mathrm{H}_{2} \mathrm{O}\right]_{4}\left[\mathrm{Cl}_{8} \cdot 12 \mathrm{H}_{2} \mathrm{O}\right]$, where the cation is an octavalent tetramer complex $\left[\mathrm{Zr}(\mathrm{OH})_{2} \cdot 4 \mathrm{H}_{2} \mathrm{O}\right]_{4}{ }^{8+}$ that can form multiple physical bonds with polyelectrolytes. ${ }^{20,22,23}$ Addition of this ion results in physical crosslinking of the percolated PBDT network. The PBDT/PAAm DN gels exhibited high modulus (up to $~ 5 \mathrm{MPa}$ ) along with toughness (work of extension up to 17.3 $\mathrm{MJ} / \mathrm{m}^{3}$ ), matching or exceeding the best DN gels. ${ }^{14,24}$ These physical DN gels also exhibited up to $51 \%$ recovery of hysteresis upon unloading, up to strains of $50 \%$.

Understanding the importance of anisotropy in biomaterials applications, researchers have recently focused on developing anisotropic hydrogels. A recent review by Sano, Ishida, and Aida discusses the depth of the approaches attempted, and can be viewed for a complete list of references. ${ }^{25}$ Hydrogels with anisotropic properties have been achieved through four primary methods: orientation of nanofillers, ${ }^{26-32}$ orientation of the polymer chain network, ${ }^{26,33,34}$ incorporation of oriented channels, ${ }^{35,36}$ and macroscale composite structures. ${ }^{37-41}$ One of the most successful techniques used to create anisotropic materials involves the introduction of bilayer structures that can be polymerized after orientation through shear flow. ${ }^{29-32}$ As a result of the incorporated oriented network, these materials exhibit one-dimensional swelling, anisotropic 
modulus, and fast-switching structural color. In some circumstances, the delineation between oriented nanofillers and oriented polymer chain structures is not so clear, as the nanofillers can form large-order domains. For example, researchers have made anisotropic, tough biomaterials through incorporating fillers (such as cellulose, collagen, and graphene oxide) that form long-range, oriented phases through stretching, ${ }^{42,43}$ drying in confined conditions, ${ }^{44}$ ionic diffusion, ${ }^{45}$ and interfacial welding. ${ }^{46}$

In this work, we aim to create anisotropic PBDT/PAAm double network hydrogels based on a physical sacrificial network of ionic-crosslinked semi-rigid polyelectrolytes. Compared to traditional DN gels that utilize swelling to extend the chains of the sacrificial first network, PBDT is inherently rigid due to its long persistence length and can be incorporated in a one-step fabrication process that does not rely upon swelling. Due to its much larger contour length in comparison to the mesh-size of the PAAm network, the linear PBDT chains are physically trapped in the PAAm matrix. Mechanical force can therefore be used to align the PBDT chains, by stretching the PAAm hydrogel without showing relaxation of the PBDT chains to the isotropic state. The oriented PBDT chains are subsequently crosslinked by immersion of the sample in $\mathrm{ZrCl}_{2} \mathrm{O}$ solution to fix the anisotropy. After the release of the mechanical force, the new geometry is maintained. The anisotropy is first visualized through optical birefringence experiments, where birefringence increases linearly with pre-stretch. X-ray scattering is next performed to quantify the ordering of the sacrificial network. We see a characteristic peak at a scattering vector of $q=0.21$ $\mathrm{nm}^{-1}$, corresponding to $30 \mathrm{~nm}$, in accordance with the persistence length of the PBDT. Integrating azimuthally, we quantify the orientation due to an increase in scattering at $0 / 180^{\circ}$ versus $90 / 270^{\circ}$. Comparing the scattering intensity in the peak and valley regions results in an anisotropy factor. After analyzing the network structure, we characterized the mechanical properties as a function of 
different pre-stretch ratios, both parallel and perpendicular to the stretching direction. Young's modulus ranging from 0.45 MPa to 1.3 MPa, and fracture stress ranging from $0.92 \mathrm{MPa}$ to 2.2 MPa is achieved, simply by modifying the architecture of the sacrificial network. Finally, we compare the structure anisotropy from x-ray scattering to the mechanical anisotropy, as a function of pre-stretch. We see a strong correlation between structural and mechanical anisotropy factors for modulus and fracture stress, demonstrating the role of the oriented physical sacrificial network on the resulting mechanical properties. The techniques demonstrated here allow us to develop anisotropic hydrogels with significantly enhanced mechanical properties compared to simple hydrogels. These materials possess properties that may help guide the design of new anisotropic materials for biological prosthetic applications. 


\section{RESULTS AND DISCUSSION}

\section{Properties of Isotropic PBDT/PAAm DN Gels}

$\mathbf{a}$

(i) Semi-rigid polymer

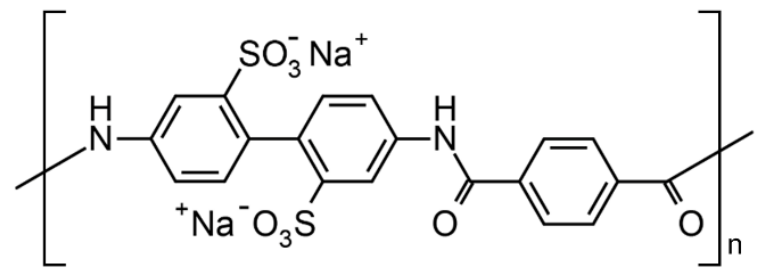

PBDT (ii) Stretchable polymer<smiles>CC(C)(C)CC(C(N)=O)C(C)(C)C</smiles>

PAAm

b

(i) Preparation

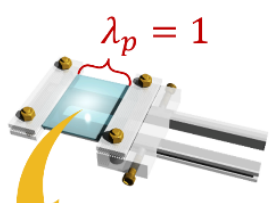

PBDT/PAAm gel (ii) $\underline{\text { Stretch }}$

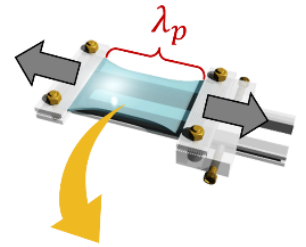

As-prepared gel

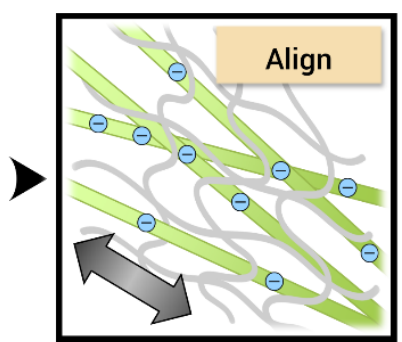

Pre-stretching (iii) Immerse

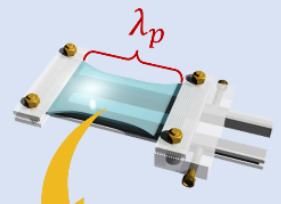

In salt sol.

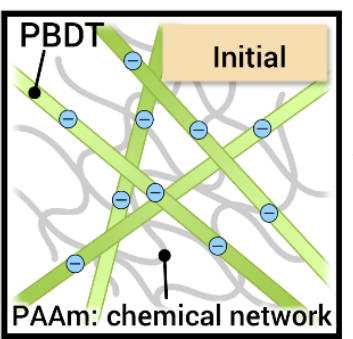

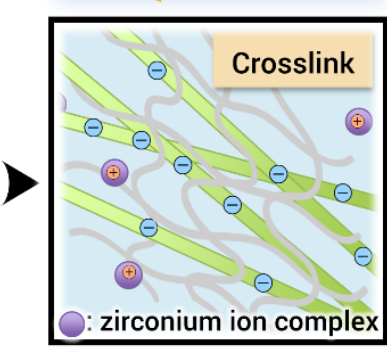

Diffusion of ions (iv) Release
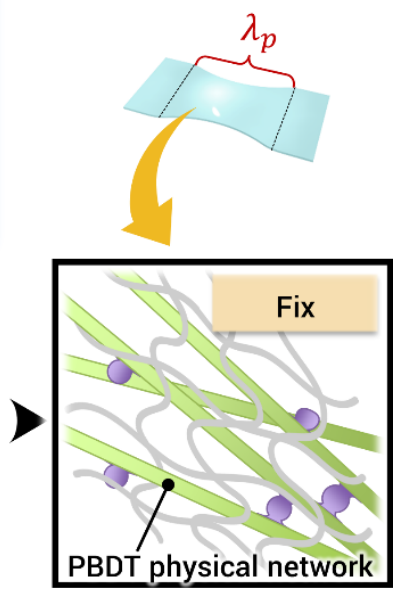

Anisotropic DN gel

Figure 1. Chemical components, and fabrication method of an oriented PBDT/PAAm DN hydrogel. (a) (i) The structure of the reinforcing PBDT, and (ii) the structure of the PAAm network. (b) The method to fabricate the oriented PBDT network. (i) The PBDT/PAAm gel sample is placed into a custom design jig and clamped. (ii) Pre-stretch is applied to the gel, to orient the PBDT chains. (iii) The sample is then immersed in $\mathrm{ZrCl}_{2} \mathrm{O}$ solution, to physically crosslink the PBDT chains. Upon release (iv), the sample maintains its shape.

To focus our investigation on the role of the sacrificial network orientation, a standard formulation will be utilized throughout all experiments. PBDT/PAAm DN gels can be tuned through a number of parameters, including crosslinking ion type, crosslinking ion concentration, and PBDT concentration. In our previous work, we have investigated these parameters in order to optimize the mechanical properties of the gel. ${ }^{15}$ In all cases, a $3.0 \mathrm{M}$ as-prepared polyacrylamide hydrogel was synthesized as the stretchable polymer network (see experimental section for full synthesis 
details). We utilize $\mathrm{ZrCl}_{2} \mathrm{O}$ as the crosslinking agent, as it can form strong crosslinks with the anionic PBDT. The concentration of the crosslinking solution is important because if it is too low, not enough crosslinks are formed, and if it is too high, intermolecular interactions are shielded. A concentration of $0.15 \mathrm{~m}$ allows for significant crosslinking to occur. Finally, it was previously shown that the overlap concentration at equilibrium conditions, $C^{*}$, was $0.3 \mathrm{wt} \%,{ }^{47}$ while the critical nematic liquid crystalline concentration, $C_{L C^{*}}$, was $2.2 \mathrm{wt} \%$, for PBDT with $\mathrm{M}_{\mathrm{w}}=182,000$ Da, similar to the $\mathrm{M}_{\mathrm{w}}=142,000$ Da PBDT used in this research. ${ }^{16}$ Between these two equilibrium concentration bounds, the PBDT can form a percolated network, without coordinated motion, which allows for highly transparent yet mechanically tough gels. A 3 wt\% PBDT prepolymer solution results in a gel containing $1.8 \mathrm{wt} \%$ PBDT at equilibrium conditions. When these parameters are combined, the produced PBDT/PAAm DN gel contains $81 \mathrm{wt} \%$ water and has $83 \%$ transparency at $550 \mathrm{~nm}$, along with a Young's modulus of 1.69 MPa, fracture stress of $1.34 \mathrm{MPa}$, fracture strain of 7.29, and an average work of extension of $5.94 \mathrm{MJ} / \mathrm{m}^{3} .{ }^{15}$

\section{Utilizing Tensile Stress to Make Anisotropic Sacrificial Networks}

The topological structure of the PBDT network within the PBDT/PAAm DN gels can be tuned by using a pre-stretching method. We have recently demonstrated that when hydrogels are loaded with stiff macromolecules and dried in confined conditions, the reinforcing phase can be oriented. ${ }^{44}$ Similarly, we can orient the PBDT molecules embedded in the gel matrix by applying an external force (e.g. stretching). ${ }^{48}$ The as-prepared PBDT/PAAm gels are placed into a custombuilt jig, that enables the application of controlled pre-stretch, as shown in Figure 1b(i). A given pre-stretch, $\lambda_{\mathrm{p}}$, is then applied and held constant (Figure $\mathbf{1 b}(\mathbf{i i})$ ). While maintaining the stretched position, the gels were submerged in $\mathrm{ZrCl}_{2} \mathrm{O}$ solution (Figure 1b(iii)) to fix the oriented PBDT molecules during the swelling process. Upon releasing from the jig, the sample geometry, with a 
new length proportional to $\lambda_{\mathrm{p}}$ was maintained even after swelling (Figure $\mathbf{1 b}(\mathbf{i v})$ ). Due to the high stiffness of PBDT and its strong crosslinking with $\mathrm{ZrCl}_{2} \mathrm{O}$, the newly formed sacrificial network causes the resulting DN gel to maintain the geometry formed from pre-stretching; in other words, the sample length does not change when released from the pre-stretching jig.

The method employed here differs from previous methods used to make anisotropic hydrogels. First, the PBDT used for reinforcement is inherently rigid and has a long contour length due to its high molecular weight $\left(\mathrm{M}_{\mathrm{w}}=142,000 \mathrm{Da}\right)$. During pre-stretching, the chemically crosslinked PAAm network cannot relax. The linear PBDT chains, although only physically embedded in the PAAm matrix, also do not relax in a relatively short time because the contour length of the PBDT chains $\left(\mathrm{L}_{\text {contour }}=\sim 460 \mathrm{~nm}\right.$ ) is much longer than the mesh size of the PAAm network $\left(\mathrm{L}_{\text {mesh }}=\sim 9 \mathrm{~nm}\right), \mathrm{L}_{\text {contour }} / \mathrm{L}_{\text {mesh }} \approx 51$ (see Supporting Information Appendix A and Figure S1 for calculations). Accordingly, the orientation of the PBDT is fixed by $\mathrm{ZrCl}_{2} \mathrm{O}$ that physically crosslinks the PBDT to form the network, and the time of physical crosslinking is governed by the diffusion of the $\mathrm{ZrCl}_{2} \mathrm{O}$ ions into the polymer network, which is faster than the relaxation time of PBDT in the PAAm matrix.

We successfully introduced anisotropic PBDT structures with different orientation degrees that were controlled by the pre-stretching ratio ( $\lambda_{\mathrm{p}}$, stretch length / initial length). The anisotropic structure was observed in the swollen samples under cross nicols (Figure 2a, black background) with $1 / 4 \lambda$ plates (Figure 2a, grey background). ${ }^{49}$ At $\lambda_{\mathrm{p}}=1.0$ (unstretched), the PBDT/PAAm DN gel did not show birefringence, indicating an isotropic structure. However, vivid birefringence was observed due to the presence of anisotropic structures at $\lambda_{\mathrm{p}}=1.5-2.5$. According to the color images under the cross nicols with a $1 / 4 \lambda$ plate, we found that the PBDT molecules were aligned along the pre-stretch direction. As $\lambda_{\mathrm{p}}$ increases, the measured birefringence also increases (Figure 2b) 
in a linear fashion.
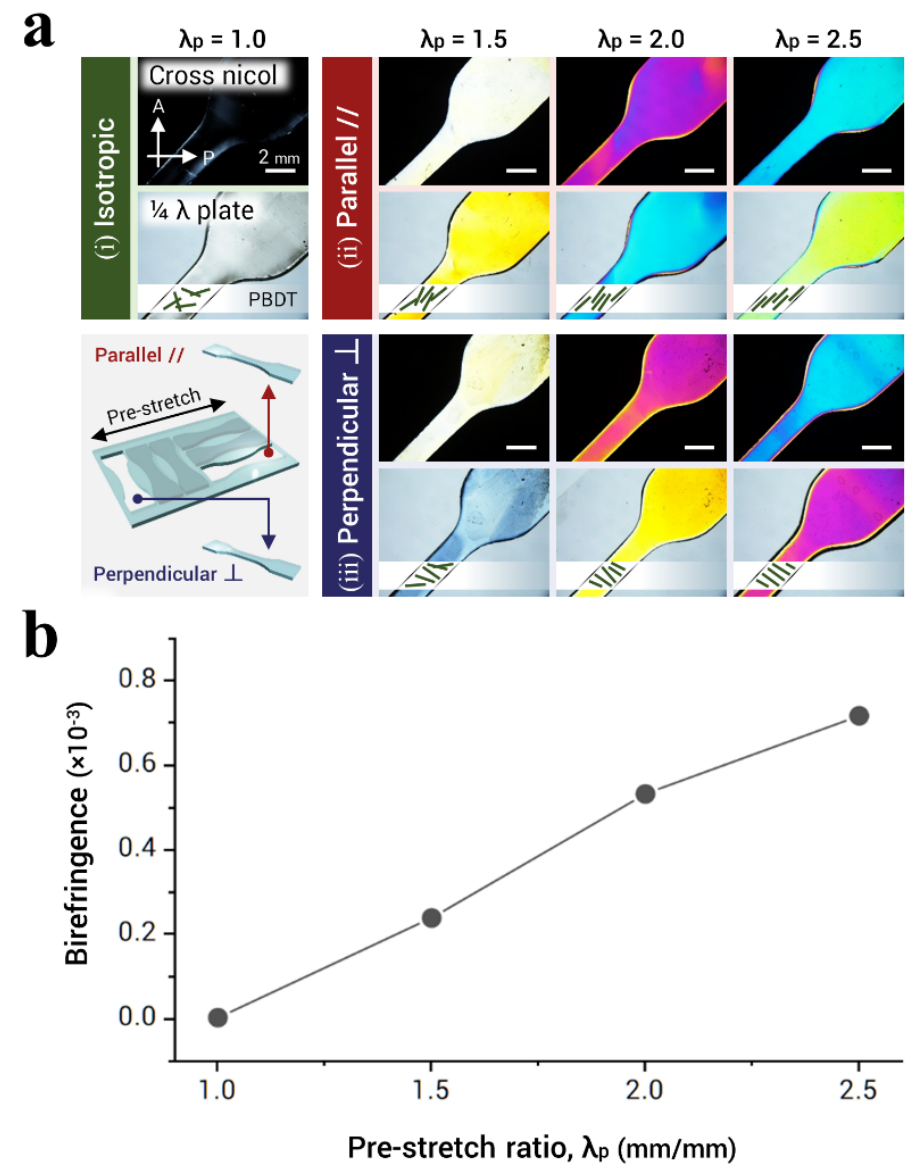

Figure 2. Orientation of the sacrificial network as confirmed by birefringence tests. (A). Images of isotropic, parallel oriented, and perpendicular oriented samples, under both cross nicols (black background), and $1 / 4 \lambda$ wave plates (grey background). (b) The measured birefringence as a function of pre-stretch ratio, $\lambda_{\mathrm{p}}$.

\section{Quantifying Anisotropy with Small Angle X-ray Scattering (SAXS)}

To further characterize the anisotropy of the gel due to the oriented PBDT structure, small angle x-ray scattering (SAXS) experiments were performed on the DN gels with various degrees of prestretch (Figure 3). As $\lambda_{\mathrm{p}}$ of the sample increased, the scattering pattern transforms from radially symmetric to noticeably oriented (Figure 3a). To analyze the orientation of the sacrificial network, 1D integration in the azimuth direction against the scattering vector, $q$, was performed, with the region between $70-110^{\circ}$ considered “perpendicular" scattering and 160-200 considered “parallel” 
a

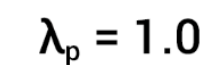

$\lambda_{\mathrm{p}}=1.5$

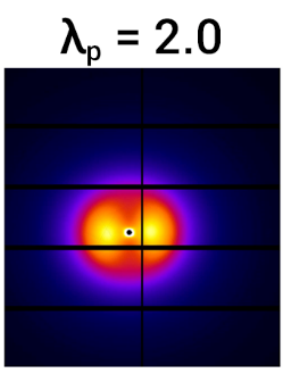

$\lambda_{\mathrm{p}}=2.5$

Pre-stretch $90^{\circ}$
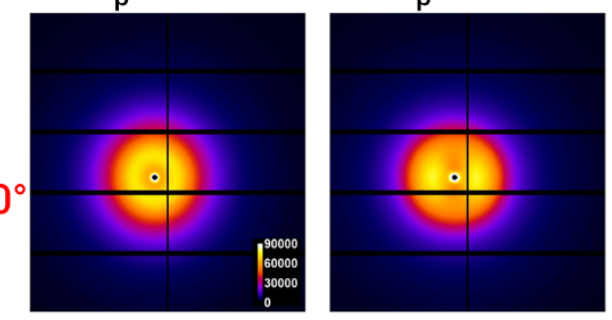

- isotropic

anisotropic
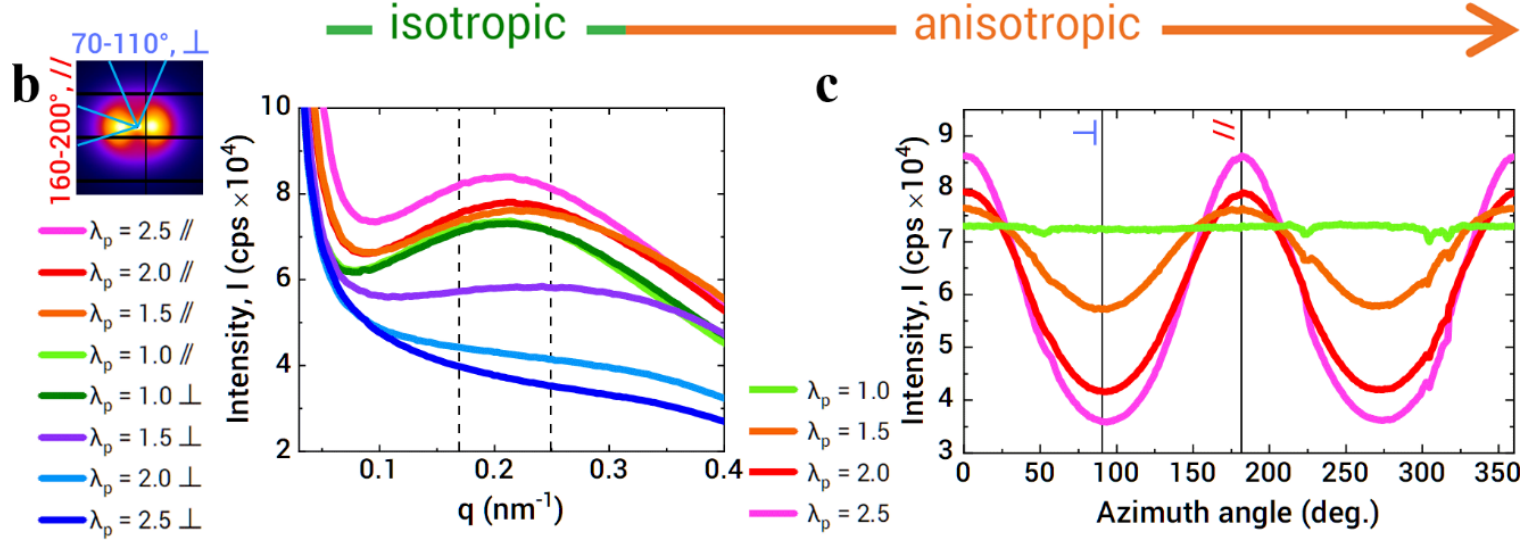

Figure 3. Small Angle X-ray Scattering (SAXS) experiments, performed on PBDT/PAAm samples with anisotropic sacrificial networks. (a) Scattering profile as a function of pre-stretch, $\lambda_{\mathrm{p}}$. As pre-stretch increased, the shape changes from radially symmetric to a two-lobed shape. (b) Intensity as a function of $q$. Intensity is higher in the parallel direction than the perpendicular direction. Dashed lines denote $q=0.17 \mathrm{~nm}^{-1}$ and $0.25 \mathrm{~nm}^{-}$ 1 , the region over which is integrated to form the plot in (c). (c) Intensity versus azimuth angle. The maximum scattering occurred near 0/180 degrees, and the minimum scattering intensity occurred near 90/270 degrees. The ratio of maximum intensity to minimum intensity was calculated as the anisotropy factor.

scattering. The intensity vs. $q$ plot for these regions with different pre-stretch ratios can be seen in

Figure 3b. A characteristic peak $\left(q=0.21 \mathrm{~nm}^{-1}\right)$ corresponding to a length of about $30 \mathrm{~nm}$ is expected to represent the persistence length of PBDT (close to the literature value of $\sim 40 \mathrm{~nm}$ for $180 \mathrm{kDa}$ PBDT). ${ }^{16}$ With increasing pre-stretch, more scattering occurs in the parallel direction than in the perpendicular direction. To better analyze this trend, we integrated the intensity between $q$ $=0.17$ and $0.25 \mathrm{~nm}^{-1}$, denoted by the dashed lines in Figure 3b, for the entire azimuthal range. The intensity versus azimuth angle plot is shown in Figure 3c. As pre-stretch increases, full width at half maximum decreases and intensity increases in the parallel direction and decreases in the perpendicular direction, indicating the long axis of PBDT aligns in the pre-stretch direction.

\section{Mechanical Characterization of Anisotropic PBDT/PAAm DN Gels.}

To investigate the orientation effect of PBDT on the mechanical properties, we performed uniaxial 
tensile tests on the anisotropic PBDT/PAAm DN gels. Large sheets of PBDT-containing PAAm gel were made, pre-stretched, and immersed in $0.15 \mathrm{M} \mathrm{ZrCl}_{2} \mathrm{O}$ solution to fix the anisotropic physical sacrificial network. After fixing the anisotropic structure, dumbbell shaped samples were punched parallel and perpendicular to the stretching direction. An isotropic $\left(\lambda_{p}=1\right)$ sample was also prepared as a control. The resulting stress versus strain curves can be seen in Figure 4a. In the case of the samples that have parallel PBDT orientation in relation to the tensile direction, the gels became harder and stronger than the control sample with increasing orientation degree of PBDT.

A noticeable change in the stress versus strain response occurs after the yielding point. In the case of the parallel samples, noticeable stiffening occurs compared to the isotropic and perpendicular-oriented samples. In the case of traditional double network gels, covalent bonds are continuously fracturing during yielding. Once a sacrificial bond is extinguished, it cannot be reformed. In the case of PBDT/PAAm DN gels, the sacrificial bonds are formed from ionic interactions, and can reform after fracture. The impact this reformation process has depends on the orientation. In the case of the parallel oriented samples, the reformation process results in significant strain-hardening during yielding. With PBDT aligned perpendicular to the loading direction however, the PBDT network supports less load during deformation, reducing the impact of this effect.

Figure 4b through Figure 4e compare the resulting properties extracted from the stress versus strain curves. Circle symbols denote parallel tests, while triangle symbols represent perpendicular tests. In the case of Young's modulus, $E$, as pre-stretch increases, the modulus deviates from the isotropic modulus of $E=0.98 \mathrm{MPa}$, increasing in the stretching direction up to $E=1.29 \mathrm{MPa}$, and decreasing perpendicular to the stretching direction to $E=0.44 \mathrm{MPa}$ (Figure 
4b). Following the same trend, an $81 \%$ increase in fracture stress, $\sigma_{x}$, was measured due to orientation of the stiff network, in the parallel direction (Figure 4c). Due to pre-stretching, we see a noticeable decrease in fracture strain for the parallel samples, and only a slight change in the perpendicular samples (Figure 4d). This is likely due to pre-stretching influencing the total extensibility of the samples. In the case of the parallel samples, pre-stretching in the loading direction results in the testing specimen possessing residual tension (and therefore decreased strain at break). For perpendicular samples, we would expect the Poisson's effect during stretching to result in residual compression, and therefore increased strain at break. In reality strain at break increases only slightly. The rigid PBDT network that possesses higher modulus is oriented perpendicular to the stretching direction and therefore will exert a resistance against the contraction in this direction when the sample is stretched. As a result, the sample is less deformed in the direction perpendicular to the stretch direction than expected from an isotropic sample, and the fracture strain will not increase as expected from an isotropic sample. This effect will be discussed
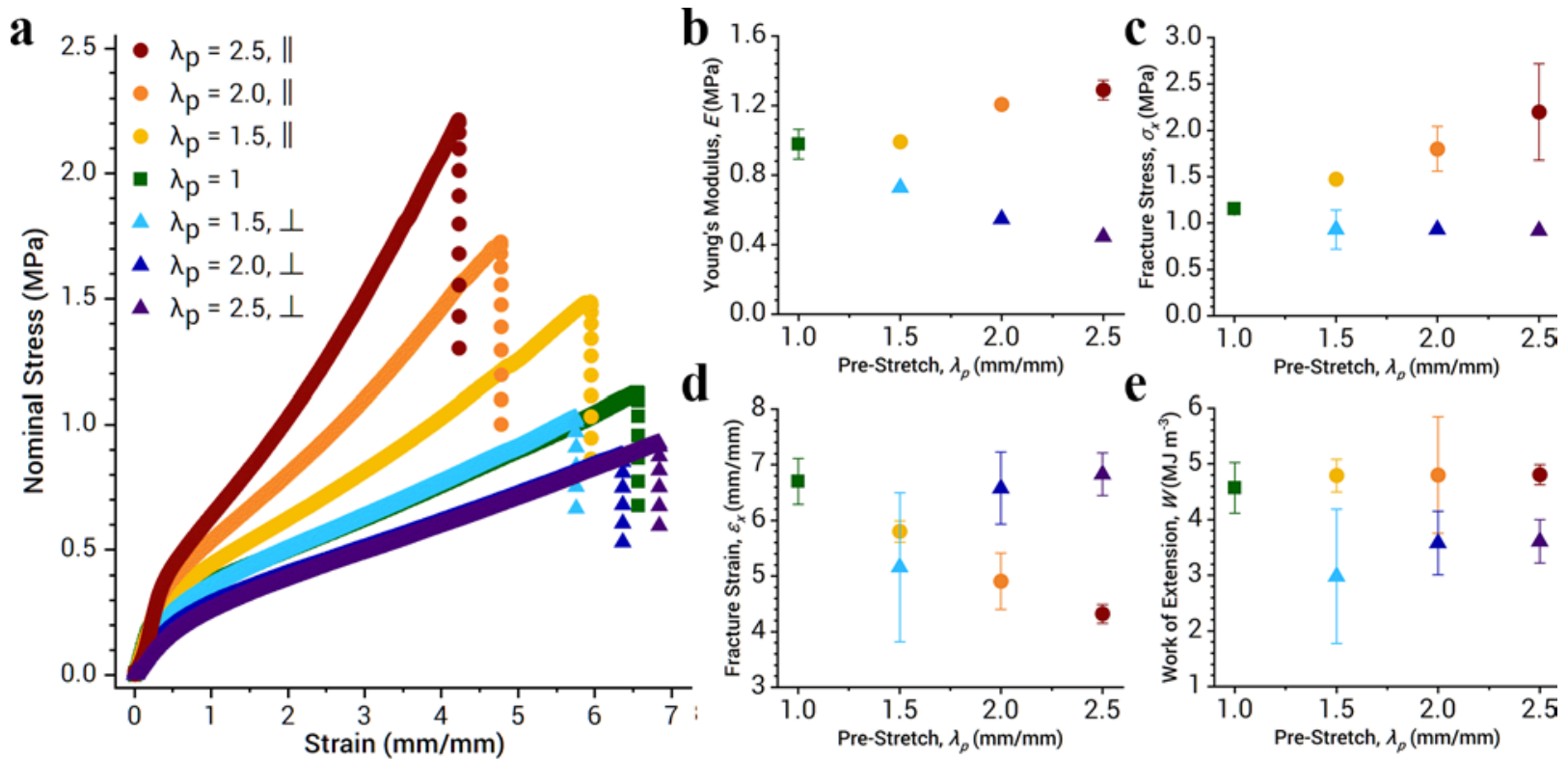

Figure 4. Mechanical response of anisotropic PBDT/PAAm DN gels. (a) Nominal stress versus strain for samples with oriented sacrificial networks. (b) Young's modulus, (c) fracture stress, (d) fracture strain, and (e) work of extension of the anisotropic PBDT/PAAm DN gels. 
further in the next section. Additionally, one sample from the $\lambda_{\mathrm{p}}=1.5$ perpendicular data set failed early, resulting in the large error bars shown. Finally, we compared the work of extension, $W$, (normalized by sample volume) of the composites, in Figure 4e. In the case of the parallel samples, despite having higher $\sigma_{x}$ they also have a lower $\varepsilon_{x}$, resulting in no significant change in $W$. The perpendicular samples show approximately the same $\varepsilon_{x}$ but lower $\sigma_{x}$, and therefore exhibit decreased $W$.

\section{Correlating Structural and Mechanical Anisotropy}

In the previous sections, we have shown that we can quantify the orientation of DN gels by x-ray scattering experiments and the anisotropic mechanical properties by tensile tests. Next, we analyze the correlations between the structural anisotropy factor of the PBDT network and the measured mechanical anisotropy factor (Figure 5). Mechanical anisotropy factor is defined as the ratio of a quantified property in the parallel direction divided by the perpendicular direction. The anisotropy factor for Young's modulus, $E$, fracture stress, $\sigma_{x}$, fracture strain, $\varepsilon_{x}$, and work of extension, $W$, are represented by the colored symbols in Figure 5a to Figure 5d, respectively. For the control sample with $\lambda_{\mathrm{p}}=1$, both the $\mathrm{x}$-ray scattering and the mechanical response are isotropic, corresponding to the anisotropy factor of 1 . Anisotropy factor for the x-ray scattering experiments was determined by dividing the maximum intensity (at an azimuth angle of 0/180 degrees) by the minimum intensity (at an azimuth angle of 90/270 degrees), as highlighted in Figure 3c. For each plot, two guidelines are drawn, as an aid to the eye: one line represents a linear response between anisotropy factor and pre-stretch where the slope, $m$, is equal to 1 , and one guideline is horizontal $(m=0)$, showing no correlation between anisotropy factor and pre-stretch.

First, we can discuss the orientation of the structure, as determined by x-ray scattering. We see that the anisotropy factor in the structure matches very closely to the line with $m=1$, meaning 
that the anisotropy factor is equal to the pre-stretching ratio. This provides good evidence that as pre-stretching increases, we are continually creating a more anisotropic sacrificial network. Next we will compare the mechanical responses to the structural anisotropy. Each plot contains the SAXS data for comparison.

For both Young's modulus (Figure 5a) and fracture stress (Figure 5b), we see a strong correlation between the increase in anisotropy factor and pre-stretch. The values are close to those measured from SAXS experiments, confirming our belief that the oriented structure results in the anisotropic mechanical response. When the PBDT becomes aligned, they formed rigid structures in the direction of pre-stretching. After the introduction of the cations, the anisotropic structures become locked. These structures result in this gel having composite properties that can be understood by simple composite averaging methods. ${ }^{50}$ When the samples are prepared with the sacrificial network aligned in the tensile direction, both components of the PBDT/PAAm DN gel experience the same strain (iso-strain limit). Since the PBDT network is stiffer than the PAAm network yet they both deform to the same degree, a significant increase in modulus is observed. On the other hand, when the sacrificial network is aligned perpendicular to the tensile direction, each component will experience the same stress (iso-stress limit). Due to its higher stiffness, the PBDT deforms much less than the PAAm network to achieve the same stress, and thus the mechanical properties are primarily governed by the matrix. Note that changing the crosslinking density of the PAAm gels by two orders of magnitude had only a small influence on modulus (Figure S2), demonstrating the small impact the stretchable network has on the ultimate mechanical properties and confirming the important role PBDT plays in controlling Young's modulus of PBDT/PAAm DN gels. By utilizing pre-stretching, we can control the architecture of the PBDT sacrificial network structure in the gel, providing significant, post-polymerization 
control over mechanical properties.

In the case of fracture strain, we see an inverse trend in mechanical anisotropy factor with increasing pre-stretch and increasing structural anisotropy: higher pre-stretch results in a decrease in strain in the stretching direction, and an increase in strain in the perpendicular direction (Figure 5c, green solid symbols). However, these results do not account for the effect of the pre-stretch on the PAAm matrix. In the case of the parallel direction, an additional stretch equivalent to $\lambda_{\mathrm{p}}$ has already been applied to the matrix prior to testing. Conversely, in the perpendicular direction, due to Poisson's effect, the network is beginning the test in a compressed state, allowing for more stretch prior to fracture. Assuming the gels are incompressible results in a decrease in true stretch
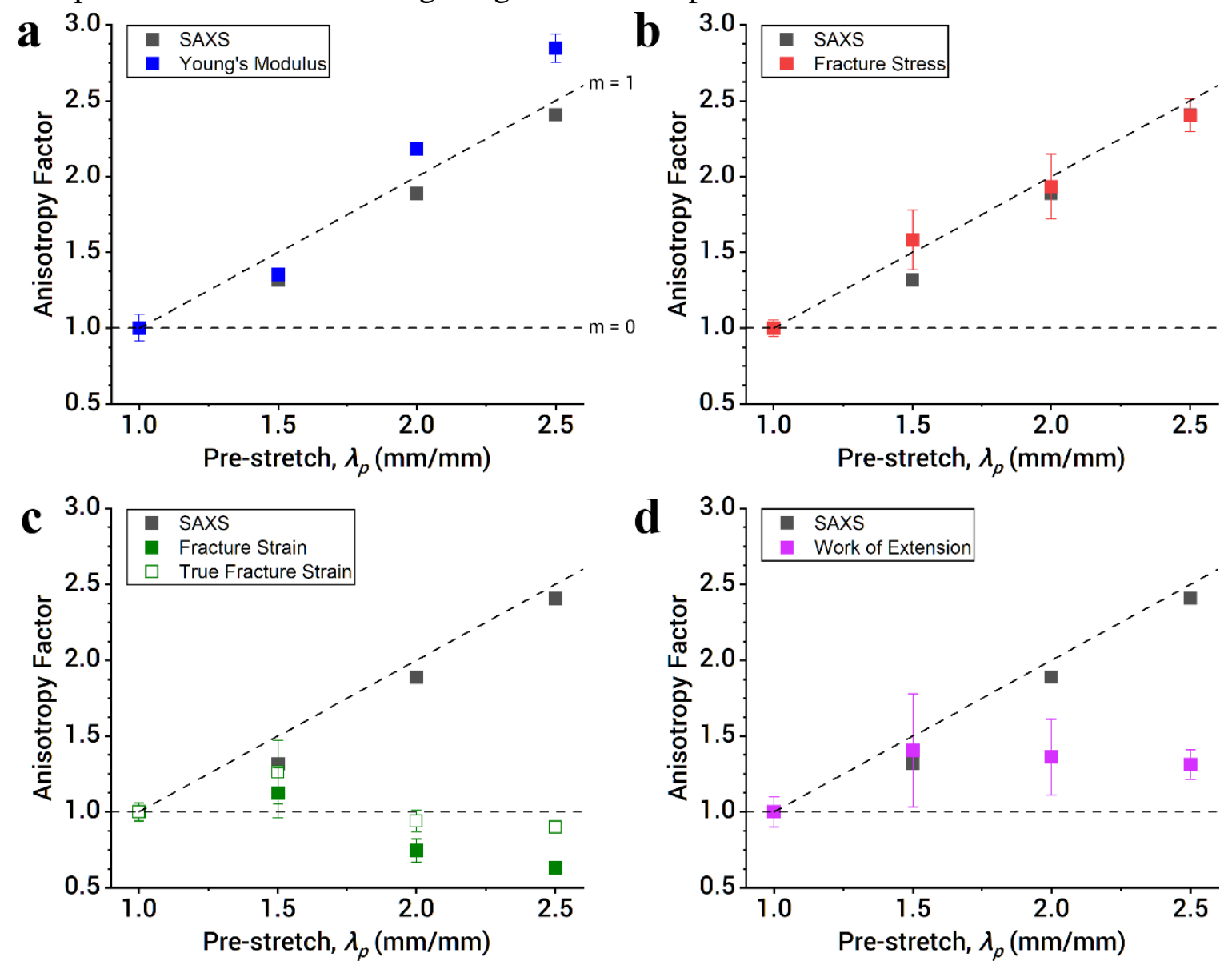

Figure 5. Comparing mechanical anisotropy to structural anisotropy. (a) Young's modulus and (b) fracture stress show strong correlations with structural anisotropy, as determined with SAXS. (c) Fracture strain shows no correlation with structural anisotropy. (d) Work of extension may show a small correlation with structural anisotropy at low pre-stretch ratios but shows no correlation in highly oriented structures. 
of $\lambda_{\mathrm{p}}{ }^{-1 / 2}$. The adjusted true strain in the parallel and perpendicular direction can be seen in Figure S3a and Figure S3b, respectively. The anisotropy factor based on this calculated true strain is shown with open symbols in Figure 5c. We now see that true fracture strain closely matches the $m=0$ guideline, representing no correlation between anisotropy factor and pre-stretch for this property. Any change in fracture strain can be directly related to the pre-stretching process and its influence on the PAAm network, not the presence of the oriented sacrificial PBDT network.

Finally, in Figure 5d, we plot the anisotropy factor of work of extension versus pre-stretch. Initially, anisotropy in work of extension matches that of the structure, but the trend quickly levels off to show no change with increasing pre-stretch. The pre-stretching process results in an oriented sacrificial network, but also results in a pre-strained PAAm matrix. At small pre-stretches, this has little influence, and optimized orientation and load-sharing within the sacrificial network results in matching anisotropy factor. However, upon further increasing pre-stretch, the pre-strained first network is limiting the total amount of energy that can be dissipated. This result demonstrates that while orienting the sacrificial network does allow us to tune the mechanical response with regards to Young's modulus and fracture stress, work of extension does not appreciably change with orientation.

\section{CONCLUSION}

Due to the high contour length and rigidity of PBDT, orientation can be induced and maintained by pre-stretching within a PAAm matrix to create robust, anisotropic physical DN gels. Immersion in multivalent $\mathrm{ZrCl}_{2} \mathrm{O}$ solution was used to fix the anisotropic structure by ionic crosslinking. Through this method, we successfully fabricated anisotropic PBDT/PAAm physical DN hydrogels. We visualized anisotropy by birefringence measurements and quantified anisotropy by small angle x-ray scattering. The degree of orientation was calculated by comparing the ratio of scattering in 
the stretching direction versus perpendicular to the stretching direction between $q=0.17 \mathrm{~nm}^{-1}$ and $0.25 \mathrm{~nm}^{-1}$, which we termed the anisotropy factor. We found that the modulus and fracture stress of the anisotropic DN gels changes proportionally with the anisotropy factor, while fracture strain and work of extension were independent of anisotropy factor. Based on this result, we can fabricate DN gels which exhibit Young's modulus and fracture stress up to 2.5x greater in the parallel direction than the perpendicular direction. The development of a method to create oriented physical sacrificial networks will help in the design of future materials for biomedical applications.

\section{EXPERIMENTAL}

\section{Materials:}

Acrylamide (AAm) (Jundei Chemical Co., Ltd) was recrystallized from chloroform. N,N’Methylenebis (acrylamide) (MBAA; Tokyo Kasei Co., Ltd.), as a crosslinker for AAm-based gels was recrystallized from ethanol. Potassium Persulfate (KPS) (Wako Pure Chemical Industries, Ltd.), as a thermal initiator for the polymerization, was used as received. Poly(2,2'-disulfonyl4,4'-benzidine terephthalamide) (PBDT), a water soluble, anionic, semi-rigid polymer, was synthesized by an interfacial polycondensation reaction. ${ }^{51}$ The synthesized PBDT had an average molecular weight, $\mathrm{M}_{\mathrm{w}}$ of about $142,000 \mathrm{Da}$, number average molecular weight, $\mathrm{M}_{\mathrm{n}}$ of about $110,000 \mathrm{Da}$, and dispersity, $\mathrm{M}_{\mathrm{w}} / \mathrm{M}_{\mathrm{n}}$ of about 1.29 . These values were determined by size exclusion chromatography (column: Shodex SB-806M) with multiple angle light scattering.

\section{Preparation of PBDT-containing PAAm hydrogels:}

The preparation of PBDT-containing PAAm hydrogels was described in detail in a previous

publication. ${ }^{15}$ Briefly, to synthesize the hydrogel sheet, reaction cells were prepared by 
sandwiching a square framed silicone spacer (thickness: $1 \mathrm{~mm}$ ) between two parallel glass plates. Aqueous pre-gel solutions containing 3.0 $\mathrm{M}$ of the neutral monomer, AAm, $3 \mathrm{wt} \%$ of the semirigid anionic polymer, PBDT, $0.1 \mathrm{~mol} \%$ of the chemical cross-linker, MBAA, and $0.5 \mathrm{~mol} \%$ of the thermal-initiator, KPS (mol\% is relative to the monomer) were prepared. After mixing, the pre-gel solution was injected into the reaction cells. Thermally induced free radical polymerization was performed in air in a temperature-controlled heat box $\left(60^{\circ} \mathrm{C}\right)$ for $10 \mathrm{~h}$. After polymerization, the as-prepared sheet-like gels (about $60 \times 60 \times 1.00 \mathrm{~mm}^{3}$ ) were gently removed from the reaction cells.

\section{Preparation of anisotropic physical sacrificial network:}

The as-prepared PBDT/PAAm gels were stretched in a uniaxial direction at various stretch ratios $\left(\lambda_{\mathrm{p}}=1 \sim 2.5\right)$, with a custom-built apparatus. After stretching, samples were immersed in a large amount of aqueous $\mathrm{ZrCl}_{2} \mathrm{O}$ solution for $24 \mathrm{~h}$. The concentration of the ionic solution was $0.15 \mathrm{M}$. Subsequently, the metal ion-loaded hydrogels were immersed in deionized water for $24 \mathrm{~h}$ to remove excess ions.

\section{Optical observation of PBDT orientation in hydrogel matrix:}

The birefringence of the PBDT/PAAm gels in the equilibrium swelling state after $\mathrm{ZrCl}_{2} \mathrm{O}$ ion exposure was observed with a polarizing optical microscope (POM) (Nikon, Eclipse, LV100POL) in the crossed polarization mode, at room temperature. Sample gels were placed on glass slides and observed from the top direction. All the samples of PBDT-containing PAAm gels exhibited a high order birefringence color, so a quarter-wave plate $(1 / 4 \lambda)$ was inserted to determine the orientation direction of the rod-like PBDT molecules or their fibrous bundles. While PBDT has a 
positive birefringence, PAAm has a negative birefringence. At the prescribed condition in this study, the birefringence intensity of PBDT surpasses that of PAAm. Therefore, the birefringence color observed was governed by the PBDT orientation.

\section{Mechanical testing:}

Uniaxial tensile tests were performed on swollen samples using a tensile-compressive tester (Instron 5965 type universal testing system). Samples were cut into a dumbbell shape standardized as JIS-K6251-7 sizes (length $35 \mathrm{~mm}$, width $6 \mathrm{~mm}$, thickness 1.5-3, gauge length 12mm, inner width 2mm) with a gel cutting machine (Dumb Bell Co., Ltd). All the samples were stretched along the length direction of the samples at an extension rate of $100 \mathrm{~mm} / \mathrm{min}$. Tensile stretch ratio, $\lambda$, is defined as $l / l_{0}$, where $l_{0}$ and $l$ are the length of the gel before and during elongation, respectively. The fracture stress and the fracture strain were defined as the nominal stress and strain at breaking point, respectively. Young's modulus was defined as slope of initial stress-strain curves. Work of extension at fracture was defined as the area under the stress-strain curve.

\section{Small Angle X-ray Scattering (SAXS):}

SAXS measurements were performed on swollen samples using the high-brightness synchrotron x-ray facility of beam line BL05XU in SPring-8 (Hyogo, Japan). The extraction wavelength of synchrotron X-ray was $1 \AA$ (12.4 keV in energy). The distance between sample and detector (Dectris Ltd., PILATUS3 S 1M) was $3.86 \mathrm{~m}$. The irradiation time was $20 \mathrm{~s}$ to obtain 2D scattering images. The pre-stretch axis of samples was set in perpendicular to the horizontal direction.

To evaluate the anisotropy of the PBDT structure, images were processed as follows. First, transmittance of the samples, $T_{s}$, was calculated from the X-ray beam intensity measured by ionization chamber (IC) at the sample stage, $I C_{0}$, and at the detector, $I C_{1}$. 


$$
T_{s}=\frac{\frac{\left(I C_{1}^{s}-I C_{1}^{d}\right)}{\left(I C_{1}^{a}-I C_{1}^{d}\right)}}{\frac{\left(I C_{0}^{s}-I C_{0}^{d}\right)}{\left(I C_{0}^{a}-I C_{0}^{d}\right)}}
$$

Superscripts s, $a$ and $d$ indicate the beam intensity of sample, air and dark, respectively. Then, the sample scattering image was divided by the transmittance, and air scattering was subtracted from the sample by image calculator processing on Image J (v1.49) software. In addition, to remove the influence of the thickness for the samples with various pre-stretch ratio, the thickness correction factor, $t_{c}$, determined from the Lambert-Beer law was multiplied.

$$
\begin{gathered}
I_{\text {corr }}=\left(\frac{I_{\text {sample }}}{T_{s}}-I_{\text {air }}\right) \times t_{c} \\
t_{c}=\frac{\ln T_{s, \lambda_{p}=1}}{\ln T_{s, \lambda_{p}}}
\end{gathered}
$$

1D integration was performed on corrected 2D scattering images using Fit_2D (v12.077) software. We defined the azimuthal angle, $\varphi$, as starting from the equatorial direction and proceeding counterclockwise on the 2D images. We integrated the intensities from $\varphi=70^{\circ} \sim 110^{\circ}$, and $\varphi=$ $160^{\circ} \sim 200^{\circ}$ to quantify “perpendicular” scattering and "parallel” scattering, respectively. The azimuthal angle distributions at the characteristic peaks were also integrated between $q=1.7 \mathrm{~nm}^{-}$ ${ }^{1}$ and $2.5 \mathrm{~nm}^{-1}$ using Fit_2D (v12.077) software. 


\section{SUPPORTING INFORMATION}

Appendix A: Calculation of contour length and mesh size. Figure S1: Schematic illustration of the unit length of PBDT. Figure S2: Effect of crosslinking density of the PAAm network on the mechanical properties of the PBDT/PAAm gels crosslinked in $0.15 \mathrm{M} \mathrm{ZrCl}_{2} \mathrm{O}$ solution. Figure S3: Comparison of measured strain and adjusted strain, accounting for pre-stretching.

\section{AUTHOR CONTRIBUTIONS}

The manuscript was written through contributions of all authors. All authors have given approval to the final version of the manuscript. Conceptualization, D.R.K., R.T., T.I., J.P.G.; Methodology, D.R.K., R.T., T.I., K.F., T.K.; Investigation, R.T., T.I. T.K.; Writing - Original Draft, D.R.K., R.T.; K.F.; Writing - Reviewing \& Editing, D.R.K., R.T., K.F., J.P.G.; Visualization, D.R.K., R.T., K.F.; Supervision, D.R.K., J.P.G.; Funding Acquisition, J.P.G.

\section{CONFLICT OF INTEREST STATEMENT}

The authors declare no competing financial interest.

\section{ACKNOWLEDGEMENTS}

This research was financially supported by Grant-in-Aid for Scientific Research (No. JP17H06144) and Grant-in-Aid for JSPS Fellows (No. 15J01078) from the Japan Society for the Promotion of Science (JSPS). This research was also partially funded by ImPACT Program of Council for Science, Technology and Innovation (Cabinet Office, Government of Japan). R.T. was supported by MEXT through the Program for Leading Graduate Schools (Hokkaido University “Ambitious Leader’s Program”). D.R.K. acknowledges the Hokkaido University Research Fund Program for Early Career Scientists. The Institute for Chemical Reaction Design and Discovery (ICReDD) was established by World Premier International Research Initiative (WPI), MEXT, 
Japan. 


\section{REFERENCES}

(1) Wegst, U. G. K.; Bai, H.; Saiz, E.; Tomsia, A. P.; Ritchie, R. O. Bioinspired Structural Materials. Nat. Mater. 2015, 14 (1), 23-36.

(2) Freedman, B. R.; Mooney, D. J. Biomaterials to Mimic and Heal Connective Tissues. Adv. Mater. 2019, 31 (19), 1-27.

(3) Yang, W.; Sherman, V. R.; Gludovatz, B.; Schaible, E.; Stewart, P.; Ritchie, R. O.; Meyers, M. A. On the Tear Resistance of Skin. Nat. Commun. 2015, 6, 6649.

(4) Woo, S. L.-Y.; Abramowitch, S. D.; Loh, John, C.; Musahl, V.; Wang, J. H.-C. Ligament Healing: Present Status and the Future of Functional Tissue Engineering. In Functional Tissue Engineering; 2003; pp 17-34.

(5) Seedhom, B. B. Ligament Reconstruction with Reference to the Anterior Cruciate Ligament of the Knee. In Mechanics of Human Joints: Physiology, pathophysiology, and treatment; 1993; pp 163-201.

(6) Miller, K.; Hsu, J. E.; Soslowsky, L. J. Materials in Tendon and Ligament Repair. In Comprehensive Biomaterials; 2011; pp 257-279.

(7) Sophia Fox, A. J.; Bedi, A.; Rodeo, S. A. The Basic Science of Articular Cartilage: Structure, Composition, and Function. Sport. Heal. A Multidiscip. Approach 2009, 1 (6), 461-468.

(8) Federico, S.; Herzog, W. On the Anisotropy and Inhomogeneity of Permeability in Articular Cartilage. Biomech. Model. Mechanobiol. 2008, 7 (5), 367-378.

(9) Temenoff, J. S.; Mikos, A. G. Review: Tissue Engineering for Regeneration of Articular Cartilage. Biomaterials 2000, 21 (5), 431-440.

(10) Okumura, Y.; Ito, K. The Polyrotaxane Gel: A Topological Gel by Figure-of-Eight CrossLinks. Adv. Mater. 2001, 13 (7), 485-487. 
(11) Gong, J. P.; Katsuyama, Y.; Kurokawa, T.; Osada, Y. Double-Network Hydrogels with Extremely High Mechanical Strength. Adv. Mater. 2003, 15 (14), 1155-1158.

(12) Sun, J.-Y.; Zhao, X.; Illeperuma, W. R. K.; Chaudhuri, O.; Oh, K. H.; Mooney, D. J.; Vlassak, J. J.; Suo, Z. Highly Stretchable and Tough Hydrogels. Nature 2012, 489 (7414), 133-136.

(13) Sun, T. L.; Kurokawa, T.; Kuroda, S.; Ihsan, A. Bin; Akasaki, T.; Sato, K.; Haque, M. A.; Nakajima, T.; Gong, J. P. Physical Hydrogels Composed of Polyampholytes Demonstrate High Toughness and Viscoelasticity. Nat. Mater. 2013, 12 (10), 932-937.

(14) Gong, J. P. Why Are Double Network Hydrogels so Tough? Soft Matter 2010, 6 (12), 2583.

(15) Takahashi, R.; Ikai, T.; Kurokawa, T.; King, D. R.; Gong, J. P. Double Network Hydrogels Based on Semi-Rigid Polyelectrolyte Physical Networks. J. Mater. Chem. B 2019, 7 (41), 6347-6354.

(16) Wu, Z. L.; Kurokawa, T.; Liang, S.; Furukawa, H.; Gong, J. P. Hydrogels with Cylindrically Symmetric Structure at Macroscopic Scale by Self-Assembly of Semi-Rigid Polyion Complex. J. Am. Chem. Soc. 2010, 132 (29), 10064-10069.

(17) Yang, W.; Furukawa, H.; Gong, J. P. Highly Extensible Double-Network Gels with SelfAssembling Anisotropic Structure. Adv. Mater. 2008, 20 (23), 4499-4503.

(18) Wu, Z. L.; Kurokawa, T.; Sawada, D.; Hu, J.; Furukawa, H.; Gong, J. P. Anisotropic Hydrogel from Complexation-Driven Reorientation of Semirigid Polyanion at Ca2+ Diffusion Flux Front. Macromolecules 2011, 44 (9), 3535-3541.

(19) Yang, C. H.; Wang, M. X.; Haider, H.; Yang, J. H.; Sun, J. Y.; Chen, Y. M.; Zhou, J.; Suo, Z. Strengthening Alginate/Polyacrylamide Hydrogels Using Various Multivalent Cations. ACS Appl. Mater. Interfaces 2013, 5 (21), 10418-10422.

(20) Dai, X.; Wang, J.; Teng, F.; Shao, Z.; Huang, X. Zr(IV)-Crosslinked 
Polyacrylamide/Polyanionic Cellulose Composite Hydrogels with High Strength and Unique Acid Resistance. J. Polym. Sci. Part B Polym. Phys. 2019, No. Iv, 1-11.

(21) Wang, Y.; Chen, Y.; Gao, J.; Yoon, H. G.; Jin, L.; Forsyth, M.; Dingemans, T. J.; Madsen, L. A. Highly Conductive and Thermally Stable Ion Gels with Tunable Anisotropy and Modulus. Adv. Mater. 2016, 28 (13), 2571-2578.

(22) Matsui, K.; Ohgai, M. Formation Mechanism of Hydrous-Zirconia Particles Produced by Hydrolysis of ZrOCl2 Solutions. J. Am. Ceram. Soc. 2005, 80 (8), 1949-1956.

(23) Li, L.; Al-Muntasheri, G. A.; Liang, F. A Review of Crosslinked Fracturing Fluids Prepared with Produced Water. Petroleum 2016, 2 (4), 313-323.

(24) Nakajima, T.; Sato, H.; Zhao, Y.; Kawahara, S.; Kurokawa, T.; Sugahara, K.; Gong, J. P. A Universal Molecular Stent Method to Toughen Any Hydrogels Based on Double Network Concept. Adv. Funct. Mater. 2012, 22 (21), 4426-4432.

(25) Sano, K.; Ishida, Y.; Aida, T. Synthesis of Anisotropic Hydrogels and Their Applications. Angew. Chemie Int. Ed. 2018, 57 (10), 2532-2543.

(26) Choi, S.; Kim, J. Designed Fabrication of Super-Stiff, Anisotropic Hybrid Hydrogels via Linear Remodeling of Polymer Networks and Subsequent Crosslinking. J. Mater. Chem. B 2015, 3 (8), 1479-1483.

(27) Fukao, K.; Nonoyama, T.; Kiyama, R.; Furusawa, K.; Kurokawa, T.; Nakajima, T.; Gong, J. P. Anisotropic Growth of Hydroxyapatite in Stretched Double Network Hydrogel. ACS Nano 2017, 11 (12), 12103-12110.

(28) Miyamoto, N.; Shintate, M.; Ikeda, S.; Hoshida, Y.; Yamauchi, Y.; Motokawa, R.; Annaka, M. Liquid Crystalline Inorganic Nanosheets for Facile Synthesis of Polymer Hydrogels with Anisotropies in Structure, Optical Property, Swelling/Deswelling, and Ion 
Transport/Fixation. Chem. Commun. 2013, 49 (11), 1082.

(29) Haque, M. A.; Kamita, G.; Kurokawa, T.; Tsujii, K.; Gong, J. P. Unidirectional Alignment of Lamellar Bilayer in Hydrogel: One-Dimensional Swelling, Anisotropic Modulus, and Stress/Strain Tunable Structural Color. Adv. Mater. 2010, 22 (45), 5110-5114.

(30) Haque, M. A.; Kurokawa, T.; Kamita, G.; Yue, Y.; Gong, J. P. Rapid and Reversible Tuning of Structural Color of a Hydrogel over the Entire Visible Spectrum by Mechanical Stimulation. Chem. Mater. 2011, 23 (23), 5200-5207.

(31) Haque, M. A.; Kurokawa, T.; Kamita, G.; Gong, J. P. Lamellar Bilayers as Reversible Sacrificial Bonds To Toughen Hydrogel: Hysteresis, Self-Recovery, Fatigue Resistance, and Crack Blunting. Macromolecules 2011, 44 (22), 8916-8924.

(32) Yue, Y.; Kurokawa, T.; Haque, M. A.; Nakajima, T.; Nonoyama, T.; Li, X.; Kajiwara, I.; Gong, J. P. Mechano-Actuated Ultrafast Full-Colour Switching in Layered Photonic Hydrogels. Nat. Commun. 2014, 5, 4659.

(33) Lin, P.; Zhang, T.; Wang, X.; Yu, B.; Zhou, F. Freezing Molecular Orientation under Stretch for High Mechanical Strength but Anisotropic Hydrogels. Small 2016, 12 (32), 4386-4392.

(34) Choi, S.; Choi, Y.; Kim, J. Anisotropic Hybrid Hydrogels with Superior Mechanical Properties Reminiscent of Tendons or Ligaments. Adv. Funct. Mater. 2019, 1904342, 1904342.

(35) Wu, J.; Zhao, Q.; Sun, J.; Zhou, Q. Preparation of Poly(Ethylene Glycol) Aligned Porous Cryogels Using a Unidirectional Freezing Technique. Soft Matter 2012, 8 (13), 3620.

(36) Barrow, M.; Zhang, H. Aligned Porous Stimuli-Responsive Hydrogels via Directional Freezing and Frozen UV Initiated Polymerization. Soft Matter 2013, 9 (9), 2723.

(37) King, D. R.; Sun, T. L.; Huang, Y.; Kurokawa, T.; Nonoyama, T.; Crosby, A. J.; Gong, J. P. 
Extremely Tough Composites from Fabric Reinforced Polyampholyte Hydrogels. Mater. Horizons 2015, 2 (6), 584-591.

(38) Zhalmuratova, D.; La, T.; Yu, K. T.; Szojka, A. R. A.; Andrews, S. H. J.; Adesida, A. B.; Kim, C.; Nobes, D. S.; Freed, D. H.; Chung, H. Mimicking “J-Shaped” and Anisotropic Stress-Strain Behavior of Human and Porcine Aorta by Fabric-Reinforced Elastomer Composites. ACS Appl. Mater. Interfaces 2019, acsami.9b10524.

(39) Liao, I.-C.; Moutos, F. T.; Estes, B. T.; Zhao, X.; Guilak, F. Composite Three-Dimensional Woven Scaffolds with Interpenetrating Network Hydrogels to Create Functional Synthetic Articular Cartilage. Adv. Funct. Mater. 2013, 23 (47), 5833-5839.

(40) Moutos, F. T.; Freed, L. E.; Guilak, F. A Biomimetic Three-Dimensional Woven Composite Scaffold for Functional Tissue Engineering of Cartilage. Nat. Mater. 2007, 6 (2), 162-167.

(41) Hubbard, A. M.; Cui, W.; Huang, Y.; Takahashi, R.; Dickey, M. D.; Genzer, J.; King, D. R.; Gong, J. P. Hydrogel/Elastomer Laminates Bonded via Fabric Interphases for StimuliResponsive Actuators. Matter 2019, 1-16.

(42) Ye, D.; Yang, P.; Lei, X.; Zhang, D.; Li, L.; Chang, C.; Sun, P.; Zhang, L. Robust Anisotropic Cellulose Hydrogels Fabricated via Strong Self-Aggregation Forces for Cardiomyocytes Unidirectional Growth. Chem. Mater. 2018, 30 (15), 5175-5183.

(43) Zhu, Z.; Li, Y.; Xu, H.; Peng, X.; Chen, Y. N.; Shang, C.; Zhang, Q.; Liu, J.; Wang, H. Tough and Thermosensitive Poly(N-Isopropylacrylamide)/Graphene Oxide Hydrogels with Macroscopically Oriented Liquid Crystalline Structures. ACS Appl. Mater. Interfaces 2016, 8 (24), 15637-15644.

(44) Mredha, M. T. I.; Guo, Y. Z.; Nonoyama, T.; Nakajima, T.; Kurokawa, T.; Gong, J. P. A Facile Method to Fabricate Anisotropic Hydrogels with Perfectly Aligned Hierarchical 
Fibrous Structures. Adv. Mater. 2018, 30 (9), 1704937.

(45) Mredha, M. T. I.; Tran, V. T.; Jeong, S.-G.; Seon, J.-K.; Jeon, I. A Diffusion-Driven Fabrication Technique for Anisotropic Tubular Hydrogels. Soft Matter 2018, 14 (37), 77067713.

(46) Mredha, M. T. I.; Le, H. H.; Tran, V. T.; Trtik, P.; Cui, J.; Jeon, I. Anisotropic Tough Multilayer Hydrogels with Programmable Orientation. Mater. Horizons 2019, 6 (7), 15041511.

(47) Yang, W.; Furukawa, H.; Shigekura, Y.; Shikinaka, K.; Osada, Y.; Gong, J. P. SelfAssembling Structure in Solution of a Semirigid Polyelectrolyte. Macromolecules 2008, 41 (5), 1791-1799.

(48) Takahashi, R.; Wu, Z. L.; Arifuzzaman, M.; Nonoyama, T.; Nakajima, T.; Kurokawa, T.; Gong, J. P. Control Superstructure of Rigid Polyelectrolytes in Oppositely Charged Hydrogels via Programmed Internal Stress. Nat. Commun. 2014, 5, 4490.

(49) Arifuzzaman, M.; Wu, Z. L.; Takahashi, R.; Kurokawa, T.; Nakajima, T.; Gong, J. P. Geometric and Edge Effects on Swelling-Induced Ordered Structure Formation in Polyelectrolyte Hydrogels. Macromolecules 2013, 46 (22), 9083-9090.

(50) Shackelford, J. F. Introduction to Materials Science for Engineers, 6th ed.; Pearson Prentice Hall, 2005.

(51) Sarkar, N.; Kershner, L. D. Rigid Rod Water-soluble Polymers. J. Appl. Polym. Sci. 1996, $62(2), 393-408$. 


\section{TOC FIGURE}

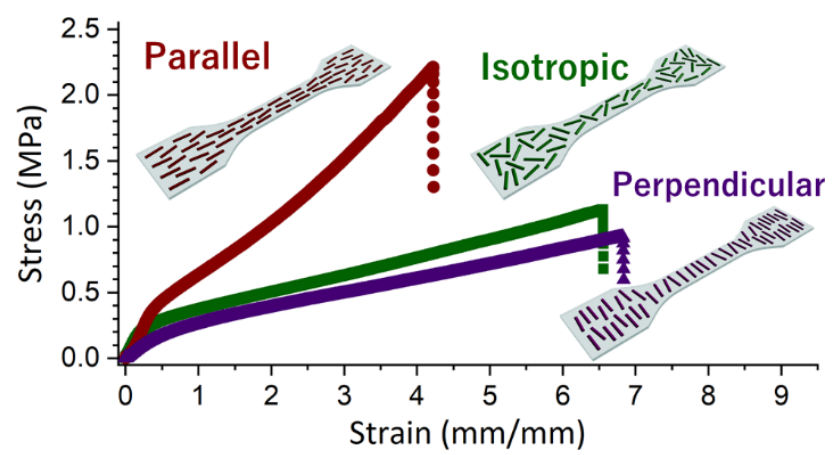

